\title{
Optimal Design of Online Measuring System for Water Content of Lubricating Oil System
}

\author{
Nan Xie ${ }^{1, a^{*}}$, Weimin Chen ${ }^{2, b}$ and Yijia Yuan ${ }^{1, c}$ \\ 'Zhejiang University of Water Resource and Electric Power, Hangzhou, China \\ ${ }^{2}$ College of Mechanical and Electrical Engineering, China Jiliang University,Hangzhou, China \\ a 595075251@qq.com, b c9419@sina.com, c905321889@qq.com
}

\begin{abstract}
Keywords: Oil water content detection; Capacitor; Neural network; Data fusion processing; Optimal design
\end{abstract}

\begin{abstract}
The measuring of water content in oil products affects the reliability and safety of the steel high speed wire rod production. Based on the existing capacitance sensor online water content detector, the neural network algorithm is used to deal with the data fusion processing with the 3D temperature experimental calibration data of the capacitance value, speed and temperature of the lubricating oil and the corresponding dielectric constant of the speed of the rolling mill in the high speed wire mill. And then, we could design optimal for capacitance lubricating oil water content detecting system. The experimental results have indicated that the method can eliminate the influence of lubrication system's air bubble and greatly improve the detection accuracy of lubricating oil water content online. Also, it has a good market promotion and application value.
\end{abstract}

\section{Introduction}

In the iron and steel production of high speed wire, wire rolling mill equipment long-term and cooling water contact, and once the lubrication system of seal ring damage there will be water penetrates the lubricating system, the metamorphism of lubricating oil emulsification, lubrication performance degradation, if not timely maintenance shutdown will lead to mechanical parts wear intensifies, causing major equipment failure, resulting in huge economic losses. Seal two kinds of doping in the application of lubrication station in iron and steel company in the high-speed wire rod mill, the rolling mill in a certain speed, gear to rotate the air and hydraulic oil interaction to produce bubbles of lubricating oil and the pipe flow, when the oil contained in the oil and gas mixture, once the hydraulic system link abrasion caused seepage, oil in the presence of oil, gas and water three mixtures based on variable medium type capacitance sensor on-line oil water content detecting instrument only to oil and water mixture were measured, the bubble will lead to the water in oil rate detection instrument to produce large error, or even lose the online detection.

Based on the existing capacitive sensor lubricating oil water content on-line detecting instrument [1-3,5-6], the detector would be optimized and designed using the neural network algorithm for detecting lubricating system data fusion processing.

\section{Bubble Growth Mechanism in the Lubrication Pipeline}

According to the theory of gas-liquid two-phase flow, there is a certain causal relationship with the growth rate of gas bubbles in the entrance velocity, lubrication system of hydraulic seal, as shown in Fig. 1, the, to rotating machinery lubrication gear, rotating machinery gear to rotate so that the gas flow rate changes with gas the form of bubbles doped into the lubricating oil, gear rotation speed of the rotating machinery more quickly, the gas flow rate is quicker, more doped into the lubricating oil in the pipeline bubble; at the same time, the lubricating oil flow in the pipeline, the gas and liquid molecules collide with each other, a part of the bubble and back into the air, when the gear speed is doped into the lubricating oil in the bubble and return to the air bubbles in maintaining a dynamic balance, so that the amount of air bubbles contained in maintaining lubricating oil In a stable value; 
when the gear, gas velocity is very small, lubricating oil produce the very small amount of bubbles, and before the gear rotation has been incorporated into the lubricating oil in the bubble is with the mixture flow and return into the air, lubricating oil contained in the amount of air bubbles is reduced gradually.

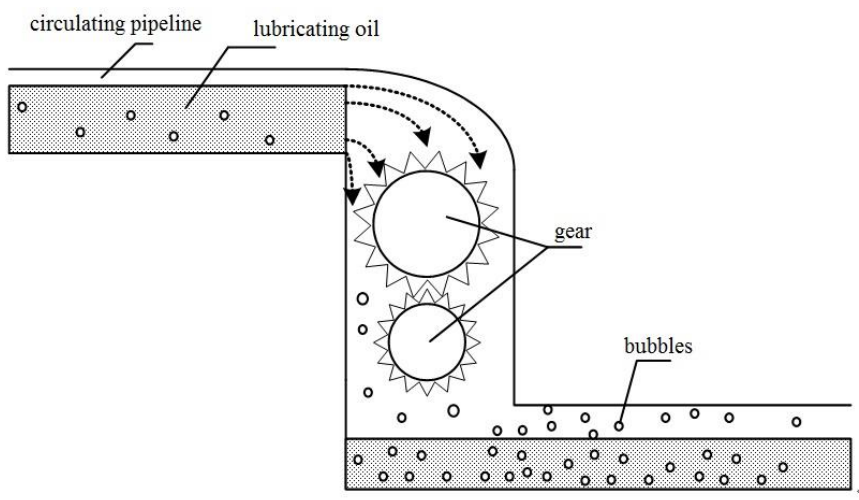

Figure 1. flow chart of lubricating oil in hydraulic circulation pipeline

\section{System Design of Online Measuring System for Water content in Oil}

As shown in Fig. 2 for hydraulic and lubrication station running water in oil rate online detection system based on, including mechanical rotational speed measuring unit, temperature sensor, capacitance sensor, serial communication, data fusion processing unit and a display unit. The rotation speed of the speed measuring unit for measuring rotating machinery, then the speed signal by serial communications transmitted to the data fusion processing unit in, temperature sensor and capacitance sensor for measuring pipe of the lubricating oil temperature and the corresponding dielectric constant capacitance value and these signals are fed to a data fusion processing unit. Data fusion processing module based on neural networks and multi - sensor data fusion of speed and size, the capacitance sensor output and the mixture temperature. Through data fusion, to eliminate the effect of air bubbles on the measurement results of output accurate measured water in oil percentage value to the display unit.

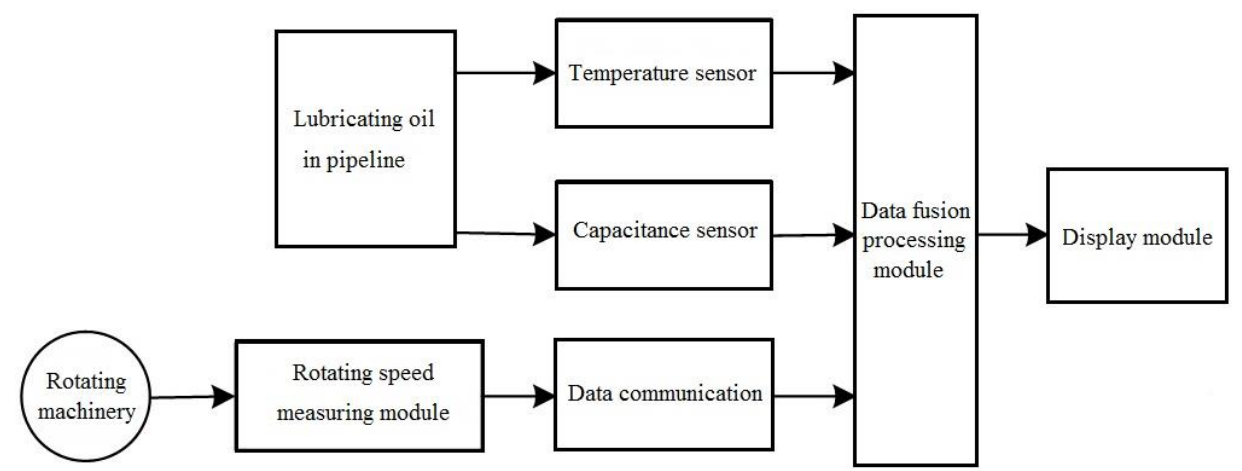

Figure 2. Design of online detection system for moisture content of oil

In the detection system, the rotating speed signals reflect the containing air bubbles in oil per oil volume, the capacitance signals reflect the dielectric constant of the mixed oil, and temperature signals reflect the oil temperature. Further, the system will establish 3D experimental calibration data about the capacitance value, speed, and temperature. The 3D experimental calibration data acquisition steps are as follows:

(1) Determinate $m$ values of water content in the range of the oil water content on-line detection system, and the corresponding capacitance values;

(2) Determine the $r$ speed values in range of the rotating machinery speed; 
(3) And then determine the q temperature values in the range of the working temperature of the oil mixture;

(4) At the last, demarcate the 3D experimental calibration data of the corresponding to the different capacitance value of $\mathrm{m}, \mathrm{r}$ and $\mathrm{q}$ about the measuring system.

\section{Data Fusion Processing Based on Multiple Sensors}

Data fusion processing module could establish artificial neural network, and the artificial neural network structure could use multilayer perceptron to the counter propagation network structure. According to 3D temperature experimental calibration data of the capacitance value, speed and temperature, we can use multi-sensor training BP neural network doing data fusion processing on each collecting speed, capacitance and temperature. Through the data fusion, it can eliminate the effect of air bubbles on the measurement results, and output accurate measured water content value of the oil [5-7].

The artificial neural network is constructed by using the forward backward propagation network structure of multi-layer perceptron. The neural network structure is shown in Fig. 3.

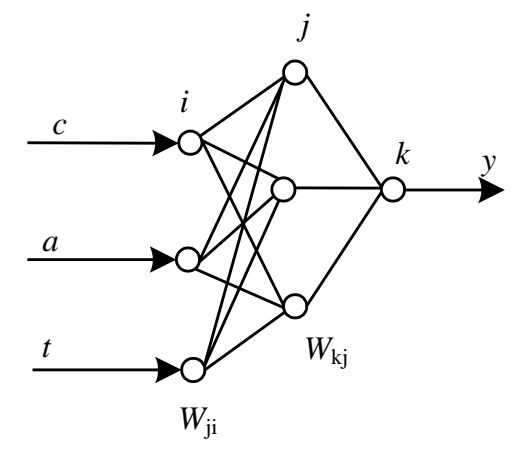

Figure 3. Neural network structure.

In Fig. 3, i, $\mathrm{j}$ and $\mathrm{k}$ are the neuron numbers of the input layer, the hidden layer and the output layer $[2,8-9]$. The neurons in the same layer are not connected with each other, and the neurons in the adjacent layers are connected with the connecting the weights of $\mathrm{Wji}$ and $\mathrm{Wkj}$. Wji is the connection weight between the input layer and the hidden layer, and the Wkj is the connection weight between the hidden layer and the output layer. The input layer has 3 nodes, the output layer has one node, and the hidden layer has 1 nodes. So the number $\mathrm{i}$ value is from 1 to 3 , the number $\mathrm{k}$ value is 1 and the number $\mathrm{j}$ is from 1 to 1 .

Neural network algorithm uses BP network training, the experimental calibration data of network training has been got as follows:

(1) Determine $m$ values of water content in the range of the oil water content on-line detection system, and measure the corresponding capacitance values;

(2) Determine the $r$ speed values in range of the rotating machinery speed;

(3) And then determine the q temperature values in the range of the working temperature of the oil mixture;

(4) At the last, demarcate the 3D experimental calibration data of the corresponding to the different capacitance value of $\mathrm{m}, \mathrm{r}$ and $\mathrm{q}$ about the measuring system.

The data fusion processing module implements the BP neural network training when the water content or temperature of the lubricating oil or the speed of the rolling mill is changed. The system gives the allowable deviation, and the output error is less than the given deviation, the output value forces near the measured target value during the network training process. Thus, the effect of bubbles and temperature on the measurement results would be greatly eliminated, after the end of the training, the output value is close to the water content of lubricating oil. 


\section{Experiment and Result Analysis}

Fig. 4 is the online monitoring curve about the water content of lubrication station of high speed wire plant of Steel Corp, and a water content online detector has been installed on each mill, each measured water content value of the online detector will be sent to the host computer monitor by RS485 communication [3-6, 10]. Fig. 4 (a) is the water content of No. 26 oil pipeline online detecting value sent to the host computer, and the detector did not use the neural network data fusion algorithm. Seen from Fig. 4 (a), when the mill is operating at a point, the measuring results of online water content detecting instrument is decreased, the final water content maintained is kept in a stable range. Therefore, the mill operation makes water content on-line detection exist a greater error on water content. Fig. 4 (b) is the water content of No. 21 oil pipeline online detecting value sent to the host computer, and the detector has adopted the neural network data fusion algorithm mentioned above. Seen from Fig. 4 (b), when the mill is operating at A point, the measuring results of online water content detecting instrument is decreased. But as the mill speed feedback signal and pipeline bubble content becomes gradually stable, the online water content detector will modify automatically. At B point, the detection results of the oil water content on-line detecting instrument are basically the same as the test results before the operation of the rolling mill. Namely, the algorithm mentioned in the paper overcomes the bubble influence of mill operation, improves the detection precision of online oil water content detecting instrument.

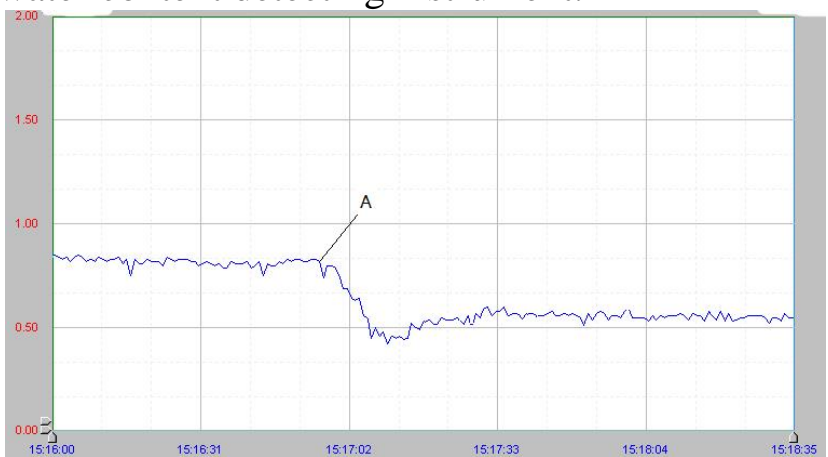

(a)

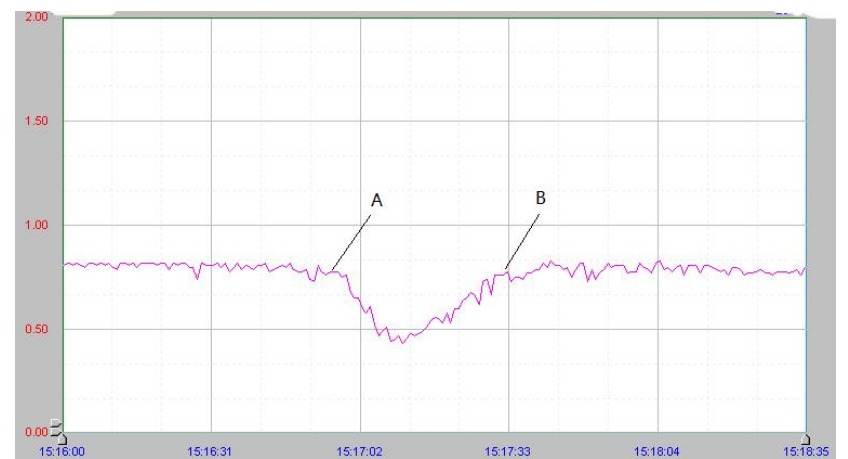

(b)

Figure 4. Comparison of two kinds of detection results

\section{Conclusion}

Based on the existing capacitive lubricating oil water content detector, authors use neural network data fusion processing on the rotating speed of the high speed wire plant of lubricating station, lubricating oil temperature and the corresponding dielectric constant capacitance value. Using this data fusion processing algorithm applied to the existing testing instruments, the detection instrument can greatly improve the detection efficiency and detection accuracy, high reliability, good repeatability, and has good promotion and application value.

\section{Acknowledgements}

This work was supported by Zhejiang Key Discipline of Instrument Science \& Technology and the 2015 New Talents Project of Zhejiang Province (Project CODE: 2015R434009).

\section{References}

[1] Hu Huimin, Gao Qian, Zhang Pingchuan. High Precision Fuel Oil Water Content Detection System Based on A R M [J]. Instrument Technique and Sensor, 2015(04):58-60. 
[2] Cheng Jianxin, Zhao Tao, Wang Zhigang. Design of signal processing circuit for micro-displacement capacitance sensing based on CDC \& ARM [J]. Transducer and Microsystem Technologies, 2013, 32(01): 105-107,111.

[3] Bian Xiaona, Liu Jing, Zhao Lizhi. Design of Capacitance Sensor's Circuit [J]. Instrument Technique and Sensor, 2008(06)

[4] Chen Weimin, Qian Xiaoyao, Hu Xinghu. Design of Online Measurement for Water-in-oil Percentage Based on Hydraulic and Lubricant System [J]. Chinese Hydraulics \& Pneumatics, 2007(11): 74-76.

[5] Chen Weimin, Qian Xiaoyao, Hu Xinghu. Design of multi-point real-time monitoring system for water-in-oil percentage [J]. Journal of China Jiliang University, 2007, 18(02): 99-102.

[6] Ding Zhenrong, Chen Weimin. New way of volume fraction measurement of water in oil based on capacitive sensor [J]. Journal of Transducer Technology, 2004, 23(05):22-24.

[7] Zhang Pingchuan, Li Xingshan, Li Buyin. Oil Water Content Detector Based on Dielectric Constant Method with High Accuracy [J]. Chinese Journal of Sensors and Actuators, 2014, 27(03): 416-420.

[8] Zhou Zhiting, Ren Xiaoxia, Feng Jianfeng. Design of intelligent detection system for moisture content of oil [J]. Advanced Measurement and Laboratory Management, 2008(06): 22-24.

[9] Cui Liqin, Qin Jianmin, Han Guangyi, etc. Capacitance Inducing Ice Thickness Sensor Based on the Relative Permittivity Difference of the Air, Ice and Water [J]. Chinese Journal Of Sensors \& Actuators. 2013, 26(01):38-42.

[10] Abdul Rajab, Aminuddin Sulaeman, Sudaryatno Sudirham. A Comparison of Dielectric Properties of Palm Oil with Mineral and Synthetic Types Insulating Liquid under Temperature Variation. ITB Journal of Engineering Science. 2011. 